\title{
Téoros
}

Revue de recherche en tourisme

\section{Un nouveau partenariat entre les personnes âgées et le tourisme}

\section{Yves Robert}

Volume 12, numéro 2, juillet 1993

La prévision-prospective du tourisme

URI : https://id.erudit.org/iderudit/1078027ar

DOI : https://doi.org/10.7202/1078027ar

Aller au sommaire du numéro

\section{Éditeur(s)}

Université du Québec à Montréal

\section{ISSN}

0712-8657 (imprimé)

1923-2705 (numérique)

Découvrir la revue

\section{Citer cet article}

Robert, Y. (1993). Un nouveau partenariat entre les personnes âgées et le tourisme. Téoros, 12(2), 52-53. https://doi.org/10.7202/1078027ar d'utilisation que vous pouvez consulter en ligne.

https://apropos.erudit.org/fr/usagers/politique-dutilisation/ 


\section{Un nouveau partenariat entre les personnes âgées et le tourisme}

Yves Robert"

Si l'on reconnaît sous le vocable personnes âgées, un groupe d'individus que les effets du vieillissement place en situation de dépendance ou de quasi dépendance de la société et de l'environnement qui est le leur, l'on doit raisonnablement souligner l'aspect relativementmarginal dece groupe et surtout le manque d'intérêt qu'il comporte pour une structure économique telle que celle du tourisme.

Par contre, si l'on accepte que le terme de personnes âgées définisse un segment de population dont les individus se trouvent en raison d'un certain âge et selon la nature des structures sociales dont ils dépendent, en situation de cessation d'activité professionnelle et bénéficiaires d'un système de retraite, force est de constater l'existence d'un potentiel réel, positif et évolutif dont le tourisme ne peut se désintéresser.

Mais l'on ne saurait se contenter de cette seule affirmation pour dresser les contours d'un marché international qui, s'il existe de manière spécifique doit répondre de la mêmemanièreà des critères qui envalident la spécificité.

Les multiples études et analyses publiées dans le domaine conservent une caractéristique commune, celle de la compilation statistique et du constat d'existence de ce segment de population à un moment déterminé, mais omettent généralement d'intégrer le paramètre vieillissement comme phénomène touchant aussi bien les jeunes que les plus âgés et créant par le renouvellement qu'il implique une chaine témoignant d'une évolution permanente, miroir d'une époque, d'une société, d'un mode de vie.

C'est à l'évidence cet élément et les attitudes qu'il mérite qui seul peut offrir à la recherche la vision certes fugitive dans le temps mais cependant bien réelle d'un groupe de oonsommateurs en général et de consommateurs de prestations tourisme en particulier.
Cependant, si l'on accepte de prendre en compte le phénomène d'allongement de la durée de la vie humaine, il est également tout à fait indispensable de considérer les changements de comportements dus à l'attitude culturelle générale et spécifique des générations actuelles qui nous sert de référence, même si cela constitue un élément perturbateur de la piste do âges sur laquelle on a tendance à cheminer.

Dans le fond, la question à laquelle nous devons répondre consiste à savoir si nous aurons soit la volonté, soit la capacité de conjuguer des paramètres autres que ceux reconnus montentents bistoriques et nous intéresser vraiment aux principaux facteurs d'influence que sont le niveau de vie et les tendances socio-démographiques.

Avec constance, les multiples études et enquêtes sur les retraitéset personnesâgées affirment, confirment et piétinent sur le faitconnu que ceux-ci ne sont plus ce qu'ils étaient.

Après tout, cela facilite peut-être l'attitude embarrassée et embarrassante des acteurs de la vie économique qui ne cessent d'hésiter devant la responsabilité qui est la leur de prendre acte de ces bouleversements et d'en tirer les conséquences. Peut-être estil délicat de se livrer ou de prêter le flancà la comparaison internationale.

Serait-il également provocateur d'extraire de source sûre le constat actuel du niveau solide de la consommation de cette catégorie de la population à comparer à celui fortement perturbé des populations en activité professionnelle et cela sans vouloir, par un artifice intellectuel que nous déplorons, engager la guerre des générations et la désamorcer dans l'heure qui suit, filon exploité actuellement au détriment de la nécessaire compréhension et complicité des uns et des autres, les uns étant en toute hypothèse appelés inexorablement à remplacer les autres.

Ceci valide en ce domaine la nécessaire analyse sociologique préalableà toutećtude prospective. C'est pour cela que nous en- 
tendons, en utilisant une constante démarche des sociétés vers une amélioration de leurs conditions de vie généraliser notre réflexion pour aujourd'hui, demain ou dans un futur plus lointain à un nombre de plus en plus importantdepays développésouen voie de le devenir et qui immanquablement devront consacrer à la satisfaction touristique une partie de plus en plus grande de leurs ressources pour une partie de leur population la plus disponible et de plus en plus à même d'exprimer le désir tourisme et loisir.

Et cette analyse va rendre dérisoire le tintamarre déclenché par les stratèges de la communication qui canonant dans les médias pensent qu'il en ressortira bien quelque chose et que leur message paré à leurs yeux de qualités exceptionnelles subjuguera la masse qu'ils n'auront alors plus qu'à recueillir,

C'est en effet faire peu de cas de tous ces constats sur l'évolution des données culturelles concernant les retraités et personnes âgées et notamment celle qui caractérise leur capacité à s'adapter aux normes d'une société qui croyait-on en avait fait des étrangers au sein de leur propre sociếté, la société dite de consommation.

C'est aussi faire peu de cas de la capacité de ces mêmes groupes de personnes de s'organiser au sein de structure représentative qui débouche bien au-delà de la tasse de thé et du jeu de carte sur des unions et fédérations actives dotées parfois directement ou indirectement de relations exceptionnelles avec une presse représentative et spécialisée parmi les plus puissantes du domaine et jouissant de la reconnaissance des pouvoirs publics et politiques.

Mais alors où se situe le point de rencontre qui permettra aux uns de goûter le plaisir d'une bonne prestation de tourisme et aux autres de comptabiliser un acte de commerce satisfaisant.

Avant d'offrir au lecteur la synthèse de notre analyse et de lui dire comment atteindre ce véritable point gáodésique, il importe de lui rappeler quelques données essentielles et notamment que les pays européens comptent quelques 90 millions de personnes concernées par notte démarche et que le pourcentage de personnes retraitées ou âgées qui voyagent par rapportau nombre totalde consommateurs de tourisme pour ces mêmes pays européens s'élève à $20 \%$ environ.

Nous inviterons également notre lecteur à se référer à l'excellent document élaboré en septembre 1992 pour la Commission Européenne du Tourisme sous le titre deA Report for the European Travel Commission, rapport qui engage un début de réponse à la question que nous traitons en rappelant que dans le domaine, le Congrès international de Cannes, organisé en 1985 par l'ETC et l'IHA sous le titre: The Senior Tourist - Solution to Seasonality a adopté 18 résolutions" toujours d'actualité.

Parmi cesrésolutions auxquelles nous avons contribué avec l'Institut de Recherche et d'Études Supérieures du Tourisme de l'Université Paris I Panthéon-Sorbonne, figure cellequi recommande l'instauration d'unvéritablepartenariatentreles différents acteurs du marché dit du tourisme retraités, à commencer par les retraités euxmêmes et les différents organismes prestataires de l'industrie du tourisme.

Et si justement les vieux nous amenaient à enterrer notre engouement moderniste pour les techniques d'in formation de masse et nous réapprenaient le dialogue, la communication etl'échange, peut-être serionsnous très proches de concevoir toutes les dimensions de ce remarquable marchéque mettent à notre portée ceux dont nous étions loin de penser qu'ils demeureraient à l'issue de leur contribution professionnelle de véritables acteurs économiques. 